\title{
Design Guidelines of a Context-Aware Notification System for Nursing
}

\author{
Masakazu Miyamae*, Futoshi Naya*, Tomoji Toriyama*, Masako Kanai-Pak ${ }^{\dagger, *}$, Kiyoshi Kogure* \\ *ATR Knowledge Science Laboratories \\ 2-2-2 Hikaridai Keihanna Science City Kyoto 619-0288 Japan \\ Email: \{miyamae, naya, toriyama, kogure\}@atr.jp \\ $\dagger$ Graduate School of Nursing, Tokyo Women's Medical University \\ 8-1, Kawada-Cho, Shinjuku-ku, Tokyo 162-8666 Japan \\ Email: kanaipak@nurs.twmu.ac.jp
}

\begin{abstract}
Providing helpful advice to nurses has great potential for decreasing medical errors. However, a notification without regard to their context may cause a medical error. To reveal the dependency between suitable information display methods and such parameters as nursing duties and the immediacy of advice, an experimental study with simulated patients was conducted in which 38 real nurses wearing an information display device on their wrists were asked to perform several nursing duties. Based on an analysis of questionnaires and the distribution of nurse response times after receiving the notification, we established guidelines for a context-aware notification system for nursing. By developing a system based on these guidelines, the system can safely display advice in a feasible and effective manner.
\end{abstract}

\section{INTRODUCTION}

Wearable computing has great potential to help workers in various enterprises. This technology is especially useful for nursing. In this study we focus on nursing because medical errors in hospitals are a serious problem [2], and providing helpful advice using wearable computers may reduce them. However, inappropriate notifications that fail to consider the nursing context may actually cause medical errors. Therefore, we investigate what parameters (e.g., nursing duties and immediacy of advice) should be used to determine a suitable notification method for implementation in a wearable system that displays advice to nurses.

In this study, based on previous work [5], we concentrated on four parameters: 1) nursing workload, 2) psychological concerns about patients, 3) immediacy of displayed advice, and 4) modality of notification. We performed an experiment to clarify the relationship between a suitable notification method and these parameters. Based on an analysis of the experiment's subjective and objective results, we established guidelines for a notification system.

\section{RELATED WORK}

Interruption or notification during work has been examined in many studies. Bailey et al. examined information display during the reading and checking of stock prices and clarified that information should be displayed in breaks between tasks [1]. Displaying information between heavy workloads should also be avoided. Using various modalities, Ho et al. examined the consequences of providing information that interrupts air traffic control procedures and argued that working efficiency improves when users can grasp the situation (e.g., priorities) of the interrupted work [3]. In wearable computing environments, Kern et al. argued that we must consider social and personal situations when displaying information [4].

Our research differs from previous efforts because we assume information display for very busy users in complicated environments and consider many factors involved in information display.

\section{EXPERIMENTS}

\section{A. Environmental Assumptions}

This study is part of the E-Nightingale project [7]. In this project, we assume environments in which various sensors are embedded in hospitals as well as nurses who also wear various sensors from which we can obtain contexts. In these environments, some necessary information (e.g., nursing duties and situations) for advising nurses is estimated from a recognition method proposed in [6]. A few examples of such advice are as follows:

- When a nurse overlooks a necessary procedure, the system gives an advice to perform it.

- When a nurse is about to start an incorrect procedure, the system gives an advice to perform it correctly.

Moreover, we assume that electronic medical charts, nursing schedules, and a database of incorrect treatments have already been prepared and that the system can receive the needed information at the appropriate times.

\section{B. Experimental parameters}

The appropriateness of information display depends on various factors. In this research we focused on the following four parameters.

1) Nursing workload: Appropriateness for nurses is reflected in the categories of nursing duties. In this experiment, we evaluated the workloads of walking and five duties.

2) Psychological concerns about patients: Nursing duties can be classified into two types: direct and indirect. In the former, nurses provide hands-on care. The latter refers to 
(a) Vital sign checks

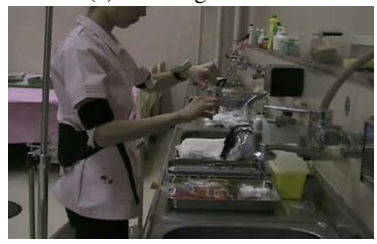

(c) Mixing medicine

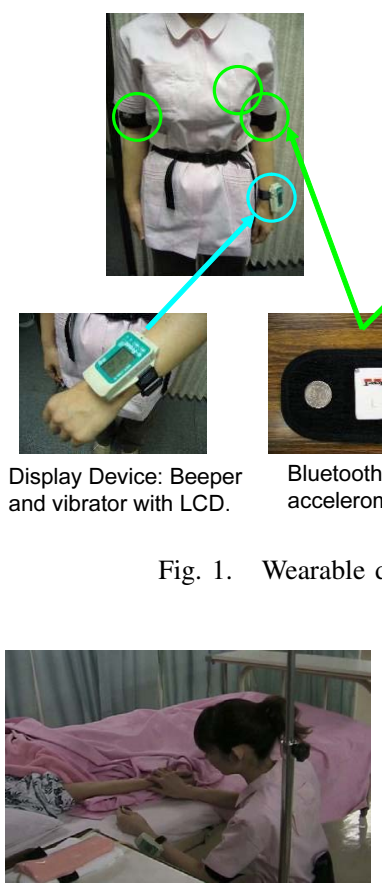

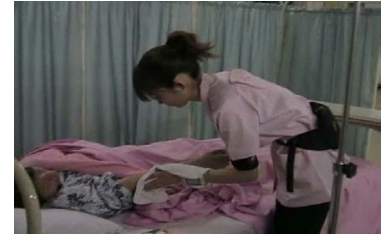

(b) Bed bath

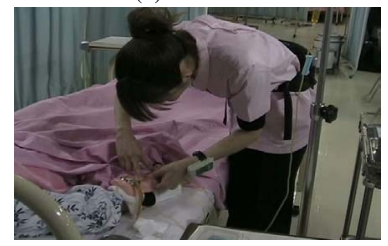

(d) Injecting a drip
Fig. 2. Snapshots of experiment

all remaining duties, including mixing medicines, writing reports, and so on. Since communication with patients is important in direct care, nurses' feelings about advice may vary by the type of duty being done.

3) Immediacy of advice: Appropriateness for nurses is also reflected in the immediacy of advice. We classified advice into two levels of immediacy and checked the response differences.

4) Notification modality: The most suitable modality depends on the current nursing task. We prepared two modalities: a vibration and/or a beeper.

\section{Experiment details}

We evaluated the relationship between these factors and information display during nursing duties at Tokyo Women's Medical University for five days during January 2007. Subjects were 38 nurses with experience ranging from 20 months to 40 years. In this experiment, subjects wore a wristwatchshaped information display device on duty (Figure 1). The device displays advice using a small liquid crystal display (LCD) and notifies users by vibration and/or a beeper. It also has a Bluetooth communication function to control display information.

The following nursing tasks were performed consecutively by the subjects in this experiment:

1) Vital sign checks: measures pulse, blood pressure, and body temperature.

2) Bed bath of the arms: wipes patient's arms with a wet towel and then with a dry towel.

3) Mixing medicine: pumps a drug solution from an ampoule with a syringe, injects it into a bottle of physiological saline, and mixes them. After that, the nurse connects a tube to the bottle and fills the tube.

4) Injecting a drip: connects a butterfly needle to the tube and injects it into the patient's arm. After that, the nurse tapes the needle and adjusts the speed of the drip.

5) Writing reports: comments on the status of patients and records performed actions.

Figure 2 shows one subject performing the tasks. Procedures that involve mixing medicine and injecting a drip impose great emotional strains because mistakes can cause serious medical errors. On the other hand, mistakes concerning vital sign checks and bed baths are far less serious. Moreover, mixing medicine and writing reports are indirect care. During the experiment, each nurse performed a sequence of the above five nursing tasks twice for different patients and could not check the tasks of other nurses. Furthermore, the experimenter recorded the tasks on video camera for later analysis. Two adult females served as patients, and we placed an imitation arm on the patient's real arm while injecting a drip. The average experimental time was about an hour per nurse.

\section{Details of displayed advice}

Advice was displayed in the middle of the above tasks and while walking. When a nurse is walking, her workload is very low. On the other hand, her workload would be high while giving injections. We selected the most inopportune time for information display to evaluate the worst case scenario. We prepared two levels of immediacy for displaying advice. An example of high-immediacy advice is "Room 603, Suzuki, time for a drip" and an example of low-immediacy advice is "Remember to wash your hands to avoid influenza." We manually sent commands to the display device using a wireless network to display these messages on the LCD and notify nurses by vibration and beeper. The following lengths and types of signals were sent to the nurses: $200 \mathrm{~ms}$ of a $2.9 \mathrm{KHz}$ beeper and/or five vibrations for high-immediacy advice and $500 \mathrm{~ms}$ of a $1.33 \mathrm{KHz}$ beeper and/or vibration once for lowimmediacy advice. Therefore, subjects could detect the degree of immediacy without monitoring the LCD.

In measurements of vital sign checks, bed baths, and writing reports, one high and one low-immediacy piece of advice were communicated by both vibration and beeper. Since we clarified that we should avoid displaying low-immediacy advice during the mixing medicine and injecting a drip tasks [5], highimmediacy advice was displayed by either vibration or beeper.

\section{EXPERIMENTAL RESULTS}

Subjects answered questionnaires about the suitable modalities of notification after every task was finished. At the end 


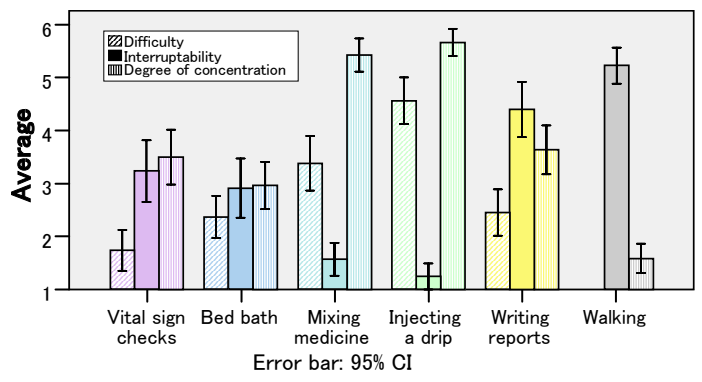

Fig. 3. Difficulty, interruptability, degree of concentration of tasks

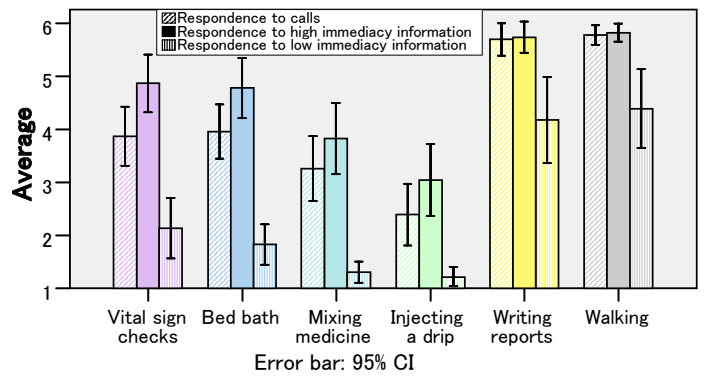

Fig. 4. Respondence to information during tasks

of this experiment they also answered questions about the characteristics of each task. Subjects provided answers on a six-point scale, with one the lowest and six the highest. In the questionnaires, we asked about the difficulty, the interruptability, and the degree of concentration during the tasks except for the difficulty of walking. If the subjects feel that they do not want to be disturbed during the task, the interruptability becomes low. The averages of the results are shown in Figure 3 . We also asked whether they responded to nurse calls or hospital mobile phones (responded to calls) and to both high and low-immediacy advice during the tasks. If the subjects feel that they will handle the information, the respondence becomes high. The results are shown in Figure 4.

We also checked the behaviors of nurses after they received the notification by analyzing captured video. Figure 5 shows the actual response time to the notification. During the task means that they checked the LCD during the highest workload situation of the task, and shortly after means they checked the LCD after completing the highest workload situation while still performing the task. After the task means that they checked the LCD after completing the task and before answering the questionnaires, and did not respond means that they did not check the LCD before answering the questionnaires. We did not display low-immediacy advice while they were performing mixing medicine and injecting a drip because we previously clarified that we should not display low-immediacy advice during such high workload tasks.

In the next section, we analyze these experimental results.

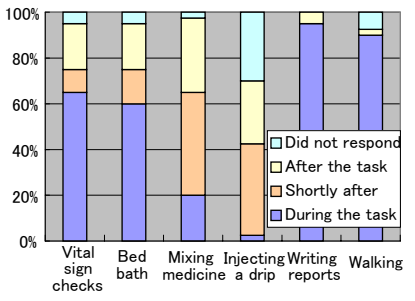

(a) High-immediacy advice

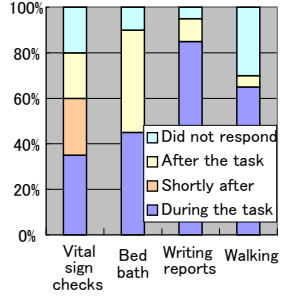

(b) Low-immediacy advice
Fig. 5. Response timing to notification

\begin{tabular}{|l|r|r|r|r|}
\hline Questions & Factor 1 & Factor 2 & Factor 3 & Factor 4 \\
\hline Writing reports - Calls & 0.901 & 0.032 & 0.121 & -0.060 \\
Walking - High immediacy & 0.900 & -0.236 & 0.012 & -0.180 \\
Walking - Calls & 0.899 & -0.096 & -0.002 & -0.114 \\
Writing reports - Low immediacy & 0.682 & 0.137 & -0.177 & 0.334 \\
Walking - Low immediacy & 0.680 & 0.300 & -0.202 & 0.246 \\
Writing reports - High immediacy & 0.662 & -0.065 & 0.391 & 0.038 \\
\hline Mixing medicine - Calls & 0.158 & 1.001 & -0.206 & 0.015 \\
Injecting a drip - Calls & -0.113 & 0.944 & -0.192 & -0.041 \\
Mixing medicine - High immediacy & 0.230 & 0.657 & 0.370 & -0.340 \\
Injecting a drip - Low immediacy & -0.087 & 0.593 & -0.030 & 0.075 \\
Injecting a drip - High immediacy & -0.222 & 0.580 & 0.337 & -0.223 \\
Mixing medicine - Low immediacy & 0.020 & 0.529 & 0.051 & -0.022 \\
Bed bath - Calls & 0.078 & 0.511 & 0.175 & 0.279 \\
\hline Bed bath - High immediacy & 0.060 & -0.074 & 0.980 & 0.169 \\
Vital sign checks - High immediacy & -0.009 & -0.037 & 0.930 & 0.139 \\
\hline Bed bath - Low immediacy & 0.124 & -0.140 & 0.044 & 0.905 \\
Vital sign checks - Low immediacy & -0.101 & 0.040 & 0.245 & 0.689 \\
Vital sign checks - Calls & -0.135 & 0.356 & 0.263 & 0.420 \\
\hline
\end{tabular}

Fig. 6. Factor analysis for interruptability

\section{ANALYSIS}

\section{A. Nursing workload}

Figure 3 shows that mixing medicine and injecting a drip are high workload tasks due to their high difficulty and degree of concentration. Moreover, the figure shows that vital sign checks and bed bath have similar tendencies and receiving notification is feasible while nurses are writing reports or walking. As described in Figure 5, the ratio for unheeded notification was especially high while injecting a drip, since they tend to ignore notification during complex and timeconsuming tasks that causes a high workload.

In addition, the interruptability graph in Figure 3 resembles the respondence graphs described given in Figures 4, and the response timing of during the task in Figure 5(a). These results suggest these are highly correlated.

\section{B. Psychological concerns about patients}

Figure 3 shows that bed bath requires less concentration but its interruptability is low. On the other hand, writing reports requires a relatively high degree of concentration but its interruptability is high. We investigated these seemingly contradictory results in post-experiment interviews with nurses and found that they were caused by nurses wanting to carefully perform direct care without being interrupted during bed baths. On the other hand, they tolerated interruption during writing reports because the task is indirect care whose priority is relatively low.

We conducted a factor analysis of the questionnaire results on responding to advice (Figure 4). Factors were extracted using least squares solutions by a promax rotation method. Four factors were extracted based on a scree plot. Figure 
6 shows its factor pattern, and we named these factors as follows: Factor 1: Monitor During Low Workload, Factor 2: Monitor Even During High Workload, Factor 3: Monitor High-Immediacy Advice During Direct Care, and Factor 4: Monitor Even Low-Immediacy Advice During Direct Care. The results show that vital sign checks and bed bath have different characteristics than other tasks despite the similar tendencies shown in Figure 4. Many subjects tended to respond to high-immediacy advice and ignore low-immediacy advice when performing the relatively easy tasks of vital sign checks and bed bath, showing that nurses do not want to be interrupted during them. This result suggests that a context-aware notification system should give attention to whether nurses are performing direct or indirect care.

\section{Immediacy of advice}

Figure 4 shows that high-immediacy advice is more important than calls. Post-experiment interviews clarified that nurses would not handle calls when busy because other nurses can handle them. Since the high-immediacy advice displayed in the experiment involved their own patients, they tended to respond to this advice because they felt directly responsible for these patients.

Figure 5 shows that they tended to respond to lowimmediacy advice later while performing vital sign checks and bed bath compared with other tasks. This result conforms to the results of Figure 4. Besides, some nurses do not respond to low-immediacy advice while walking because they do not notice it. These results also show that they can comprehend the immediacy of advice from vibration times or sound frequency even when they are performing nursing duties.

\section{Notification modality}

Since the notification modality results resemble those described in [5], we summarize them as follows.

From the analysis, most nurses prefer receiving signals by vibration while performing such direct care as vital sign checks and bed bath because they do not want to disturb patients. On the other hand, the modality preference varies among the nurses for notifications while they are performing indirect care.

\section{DESIGN PRINCIPLE}

We established the following guidelines for a notification system based on the analytical results.

1) The system should notify nurses in differentiable ways to enable them to determine whether or not they have to immediately respond to the advice.

2) The system should avoid notification when a nurse's workload is high unless the advice can prevent an imminent medical error. If it needs to display advice, the system should wait and display it when the workload decreases or display it to other nurses.

3) When a nurse is performing direct care, the system should only display emergency or important advice directly related to the present patient/task by vibration to avoid disturbing patients. The system also needs to remind nurses by using such means as blinking LEDs, since nurses tend to overlook notifications.

4) The system can display much advice when a nurse is performing indirect care and her workload is low. In this case, the system should select a notification method based on individual preference whenever possible.

To implement a context-aware notification system based on these guidelines, the system requires functions to recognize nurses' context (recognition), generate advice (generation), acquire nurses' preferences (preference), and control timing of information display (timing). Recognition recognizes and provides nurses' current context. Generation generates advice information and notifies it with its immediacy. Preference manages and provides nurses' preference information. Timing determines the best notification method based on the guidelines and information from the other functions.

By Developing a system with the above capabilities, the system is expected to safely display advice in a feasible and effective manner.

\section{CONCLUSION}

In this study, we experimentally investigated the requirements for information display and established the guidelines of a notification system for nurses. The results suggest the importance of considering the nursing workload, the psychological concerns about patients, and the immediacy of displaying advice to decide the timing and modality of notification.

In the future, we will develop a nursing advice system that displays useful advice based on these guidelines. In further experiments, we are planning to investigate oblivescence of monitoring advice. The results will contribute to the design of a suitable reminding method or a method to predict medical error caused by oblivescence.

\section{ACKNOWLEDGMENTS}

This research was supported in part by the National Institute of Information and Communication Technology. We express gratitude to all of subjects who participated in our experiment.

\section{REFERENCES}

[1] Bailey, B. P., Konstan, J. A. and Carlis, J. V.: Measuring the effects of interruptions on task performance in the user interface, SMC 2000, Vol. 2, pp. 757-762 (2000).

[2] Corrigan, J., Kohn, L. T. and Donaldson, M. S.: To Err Is Human: Building a Safer Health System, National Academies Press (1999).

[3] Ho, C.-Y., Nikolic, M. I., Waters, M. J. and Sarter, N. B.: Not now! Supporting interruption management by indicating the modality and urgency of pending tasks, Human Factors, Vol. 46, No. 3, pp. 399-409 (2004).

[4] Kern, N. and Schiele, B.: Context-Aware Notification for Wearable Computing, ISWC 2003, pp. 223-230 (2003).

[5] Miyamae, M., Naya, F., Noma, H., Toriyama, T. and Kogure, K.: A Trial Design of an Information Display Method for Medical Nursing, UbiComp 2006 Poster Session (DVD-ROM) (2006).

[6] Naya, F., Ohmura, R., Takayanagi, F., Noma, H. and Kogure, K.: Workers' Routine Activity Recognition Using Body Movements and Location Information, ISWC 2006, pp. 105-108 (2006).

[7] Noma, H., Ohmura, A., Kuwahara, N. and Kogure, K.: Wearable Sensors for Auto-Event-Recording on Medical Nursing - User Study of Ergonomic Design, ISWC 2004, pp. 8-15 (2004). 